\title{
Stability and stabilization of differential repetitive processes with time-delays over finite frequency ranges
}

\author{
Wojciech Paszke, Eric Rogers, Hongfeng Tao, Krzysztof Gałkowski
}

\begin{abstract}
This paper addresses the problem of stability and controller design for differential linear repetitive processes with time delays in state. Delay-dependent conditions for stability along the pass of such processes are developed in terms of linear matrix inequalities. These results are then extended to include finite frequency specifications to reduce conservatism generated by considering the entire frequency spectrum. The method is based on the generalized Kalman-Yakubovich-Popov (KYP) lemma and hence finite frequency range performance specifications can be imposed during the stability checking with an extension to algorithms for controller design. A simulation example to demonstrate the new results is also given.
\end{abstract}

\section{INTRODUCTION}

In the recent years, increasing research effort has been directed at the development of an applicable control systems theory for repetitive processes, which are one of the most important classes of two-dimensional (2D) systems both in terms of system theoretic properties and applications. Reported applications of these processes include long-wall coal cutting and metal rolling operations, see, e.g., the references cited in [1], [2]. Moreover, the stability theory developed for these processes can be of use in solving problems in other areas. Examples include iterative learning control (ILC) law design, with a follow through to experimental verification, see, e.g., [3] and iterative algorithms for solving nonlinear dynamic optimal control problems based on the maximum principle [4].

Repetitive processes are an example of systems that complete the same finite duration task, then resets to the starting location, makes another completion and so on. Each completion is termed a pass, its duration the pass length and the output is termed the pass profile. The notation for variables in this paper is $y_{k}(t), 0 \leq t \leq \alpha$ where $y$ is the vector or scalar-valued variable, the integer $k \geq 0$ the pass number and $\alpha<\infty$ is the pass length. Suppose also that $y_{0}(t)$ is a supplied initial pass profile and denote the sequence of pass profiles generated in response to the boundary conditions (of which $y_{0}(t)$ is a part) and/or input as $\left\{y_{k}\right\}$. Then the distinguishing feature of a repetitive process is that $y_{k}(t)$ acts as a forcing function on the next pass

W. Paszke and K. Gałkowski are with the Institute of Control and Computation Engineering,University of Zielona Góra, ul. Szafrana 2, 65-516 Zielona Góra, Poland. E-mail: \{w.paszke, k.galkowski\}@issi.uz.zgora.pl

E. Rogers is with the Department of Electronics and Computer Science, University of Southampton, Southampton SO17 1BJ, UK. Email: etarlecs.soton.ac.uk

H. Tao is with Key Laboratory of Advanced Process Control for Light Industry of Ministry of Education, Jiangnan University, Wuxi 214122, P. R. China. E-mail: taohongfeng@hotmail.com and sequence generated $\left\{y_{k}\right\}$ can contain oscillations that increase in amplitude from pass-to-pass (i.e., $k$ ) and these cannot be controlled by standard, also known as 1D in some of the literature, control laws.

Repetitive processes operate over $\{(k, t):[0, \infty) \times[0, \alpha]\}$, which is a subset of the upper-right quadrant of the $2 \mathrm{D}$ plane. They are a distinct class of $2 \mathrm{D}$ systems that operate over the complete upper-right quadrant of the $2 \mathrm{D}$ plane. If $t$ is a discrete variable then links exist with representations for 2D systems and this allows systems theory for this latter class to be applied to repetitive processes, see, e.g., [2]. However, there are repetitive process dynamics for which this is not possible. For example, the initial conditions at the start of each new pass can be an explicit function of sample instants along the previous pass. This form of dynamics cannot be represented by $2 \mathrm{D}$ systems models but just this form of pass initial conditions is required in the optimal control application [4].

The applicability of systems theory for $2 \mathrm{D}$ models is less relevant if the dynamics along the pass are governed by a matrix linear differential equation and known as differential linear repetitive processes. Also there is a need, as in other areas, to deal with the effects of time delays [5]. An example is again from the coal mining example where the pass profile on any pass can only be measured as a delayed quantity. This is because it is not possible to co-locate the measuring equipment with the cutting drum used to extract the coal.

In this paper the novel contributions are on stability and stabilization of differential linear repetitive processes with delays in the state vector. Firstly, the solution to the stability along the pass problem for processes with a single time delay are derived where the duration of the delay is include and therefore a delay-dependent result is obtained. Next, a version of the generalized Kalman-Yakubovich-Popov (KYP) lemma [6] is used to reduce the possible conservatism generated by analyzing over the complete frequency domain. Finally, the stabilization problem is solved. All results derived can be computed using Linear Matrix Inequalities (LMIs).

This paper is organized as follows: Section II gives the required background and in Section III conditions for stability along the pass are derived using the generalized KYP lemma and results in LMI constraints that ensure stability over a finite frequency range. Section IV. Section V illustrates the feasibility and effectiveness of the new results by a numerical example. Finally, the main results are summarized in Section VI together with some possible areas for further research. 
Throughout this paper, the null and identity matrices with the required dimensions are denoted by 0 and $I$, respectively, and the notation $X \prec Y$ (respectively $X \succ Y$ ) means that the matrix $X-Y$ is negative definite (respectively, positive definite). The notation $(\star)$ represents the transposed elements in some symmetric matrices and $\rho(\cdot)$ denotes the spectral radius of its matrix argument, i.e., if $\lambda_{i}, 1 \leq i \leq q$, denotes the eigenvalues of a $q \times q$ matrix, say $H, \rho(H)=\max _{1 \leq i \leq q}\left|\lambda_{i}\right|$. Also $\operatorname{sym}\{\mathrm{M}\}$ is a shorthand notation for $M+M^{T}, \otimes$ denotes the Kronecker matrix product.

The following lemma is used in developing the new results in this paper.

Lemma 1: [7] Given a symmetric matrix $\Upsilon \in \mathbb{R}^{p \times p}$ and two matrices $\Lambda, \Sigma$ of column dimension $p$, there exist a matrix $W$ such that the LMI

$$
\Upsilon+\operatorname{sym}\left\{\Lambda^{T} W \Sigma\right\} \prec 0,
$$

holds if, and only if

$$
\Lambda^{\perp^{T}} \Upsilon \Lambda^{\perp} \prec 0, \Sigma^{\perp^{T}} \Upsilon \Sigma^{\perp} \prec 0,
$$

where $\Lambda_{\perp}$ and $\Sigma_{\perp}$ are arbitrary matrices whose columns form a basis of null spaces of $\Lambda$ and $\Sigma$ respectively. Clearly, this means that $\Lambda \Lambda_{\perp}=0$ and $\Sigma \Sigma_{\perp}=0$.

\section{DifFERENTIAL LINEAR REPETITIVE PROCESSES AND THEIR STABILITY PROPERTIES}

Consider a differential linear repetitive process defined over $0 \leq t \leq \alpha$ and $k \geq 0$, by the following state-space model (see [2] for further details)

$$
\begin{aligned}
& \dot{x}_{k+1}(t)=A x_{k+1}(t)+B u_{k+1}(t)+B_{0} y_{k}(t), \\
& y_{k+1}(t)=C x_{k+1}(t)+D u_{k+1}(t)+D_{0} y_{k}(t),
\end{aligned}
$$

where the pass length is denoted by $\alpha<+\infty$ and $x_{k}(t) \in$ $\mathbb{R}^{n}, u_{k}(t) \in \mathbb{R}^{m}$ and $y_{k}(t) \in \mathbb{R}^{p}$ represent, respectively, the state, input and output vectors at time instant $t$ on pass $k$. The boundary conditions considered are

$$
\begin{aligned}
x_{k+1}(0) & =d_{k+1}, \quad k \geq 0, \\
y_{0}(t) & =f(t), \quad 0 \leq t \leq \alpha,
\end{aligned}
$$

where $d_{k+1} \in \mathbb{R}^{n}$ is the pass state initial vector with known constant entries and $f(t) \in \mathbb{R}^{m}$ is the initial pass profile vector whose entries are known functions of $t$.

The terms $B_{0} y_{k}(t)$ and $D_{0} y_{k}(t)$ in (2) describe the contributions of the previous pass profile vector to the current pass state and pass profile vectors respectively. As discussed in the previous section these terms are the source of control problem for these processes, i.e., the sequence of pass profiles $\left\{y_{k}\right\}_{k \geq 1}$ can contain oscillations that increase in amplitude from pass-to-pass. As this behavior cannot be controlled by standard linear systems control laws, a stability theory for these processes has been developed [2]. This theory requires that a bounded initial pass profile produces a bounded sequence of pass profiles, where in the strongest form this property is required for all possible values of the pass length. This last form of stability is termed stability along the pass and the following result characterizes this property.

Lemma 2: [2] A differential linear repetitive process described by (2) and (3) is stable along the pass if and only if

i) $\rho\left(D_{0}\right)<1$,

ii) all eigenvalues of the matrix $A$ have strictly negative real parts,

iii) all eigenvalues of $G(j \omega)=C(j \omega I-A)^{-1} B_{0}+D_{0}$ have modulus strictly less than unity $\forall \omega \geq 0$.

In terms of checking these conditions for a given example, it is the third condition that will incur the greatest computational load, since it requires that all eigenvalues of $G(j \omega)$, also termed the characteristic loci, lie inside the unit circle in the complex plane $\forall \omega$.

This paper considers differential linear repetitive processes with a delay in the current pass state vector and described by the state-space model

$$
\begin{aligned}
& \dot{x}_{k+1}(t)=A x_{k+1}(t)+A_{d} x_{k+1}(t-d)+B u_{k+1}(t)+B_{0} y_{k}(t), \\
& y_{k+1}(t)=C x_{k+1}(t)+C_{d} x_{k+1}(t-d)+D u_{k+1}(t)+D_{0} y_{k}(t),
\end{aligned}
$$

where $d$ is the unknown time-delay constant satisfying $0<$ $d \leq \bar{d}$, and $\bar{d}$ is a known upper bound. No loss of generality arises from assuming $x_{k}(t)=x_{0, k}, t \in[-d, 0]$ on each pass.

The following result characterizes stability along the pass of processes described by (4).

Lemma 3: [8] A differential linear repetitive process described by (4) is stable along the pass for all $d \in[0, \bar{d}]$ if and only if

i) $\rho\left(D_{0}\right)<1$,

ii) all eigenvalues of the matrix $\left(A+e^{-d j \omega} A_{d}\right)$ have strictly negative real parts $\forall \omega \geq 0$ and $d \in[0, \bar{d}]$,

iii) all eigenvalues of $G_{c}(j \omega)=\left(C+e^{-d j \omega} C_{d}\right)$ $\times\left(j \omega I-A-e^{-d j \omega} A_{d}\right)^{-1} B_{0}+D_{0}$ have modulus strictly less than unity $\forall \omega \geq 0$ and $d \in[0, \bar{d}]$.

Proof: (Sketch of the proof): The result of Lemma 3 is based on reworking the conditions of Lemma 2 and application of delay-dependent stability conditions for the state dynamics on each pass to be bounded for any set of boundary conditions. Also, the condition is modified to impose frequency attenuation (of the frequency content of the initial pass profile) over the complete spectrum for any delay $d \in[0, \bar{d}]$.

The development of a direct and numerically tractable method to check the above conditions and extend them to design procedures. An alternative, at the possible expense of some conservativeness, in developed in the next section that can be checked by solving convex optimization problem over LMIs.

\section{STABILITY ALONG THE PASS}

By Lemma 3 a differential linear repetitive process described by (4) is stable along the pass if and only if

$$
\left\|G_{c}(j \omega)^{k}\right\| \rightarrow 0, \text { as } k \rightarrow \infty, \forall \omega \geq 0,
$$

where $\left\|G_{c}(j \omega)\right\|=\sup _{0 \leq \omega \leq \infty}\left|G_{c}(j \omega)\right|$ and

$$
G_{c}(j \omega)=\left(C+e^{-d j \omega} C_{d}\right)\left(j \omega I-A-e^{-d j \omega} A_{d}\right)^{-1} B_{0}+D_{0},
$$


and hence each frequency component is attenuated from pass to pass as $k \rightarrow \infty$. Moreover, it is clear that any frequency component is reduced between successive passes if and only if

$$
\rho\left(G_{c}(j \omega)\right)<1, \forall \omega \geq 0 .
$$

Based on standard Lyapunov stability theory, routine arguments show that this last condition can be replaced by the requirement that there exists a Hermitian matrix $P(j \omega) \succ 0$ such that

$$
G_{c}(j \omega)^{*} P(j \omega) G_{c}(j \omega)-P(j \omega) \prec 0, \forall \omega \geq 0,
$$

but the dependence of $P(j \omega)$ on the frequency variable $\omega$ is unknown. To remove this difficulties, one method is to consider the use of constant real matrices over entire frequency range.

Lemma 4: Consider a differential repetitive process described by (4) with transfer-function matrix defined by (6). Then (7) holds if there exist $P \succ 0$ such that

$$
\left[\begin{array}{c}
G_{c}(j \omega) \\
I
\end{array}\right]^{T}\left[\begin{array}{cc}
P & 0 \\
0 & -P
\end{array}\right]\left[\begin{array}{c}
G_{c}(j \omega) \\
I
\end{array}\right] \prec 0, \forall \omega \geq 0 .
$$

Remark 1: This result establishes that a Hermitian matrix in this case can be replaced by real symmetric matrix $P$ (see some details in [9]) but $P$ must work for $\forall \omega \geq 0$. Moreover, it may introduce additional conservatism since it has been obtained by keeping $P_{2}$ constant and independent of frequency. However, this change reduces the computational load of the resulting conditions and allows for extension to control law design.

Practical experience shows that some examples exhibit poor transients during operating even if the above condition is satisfied (i.e., the output may grow over some number of passes). To avoid these problems, a stronger stability criteria is required and one choice is to require that

$$
\bar{\sigma}\left(G_{c}(j \omega)\right)<1, \forall \omega \geq 0
$$

where $\bar{\sigma}(\cdot)$ denotes the maximum singular value of its matrix argument. Also since

$$
\bar{\sigma}\left(G_{c}(j \omega)\right)<1 \Leftrightarrow\left\|G_{c}(j \omega)\right\|_{\infty}<1, \forall \omega \geq 0
$$

then (9) holds if (8) is feasible for $P=I$.

\section{A. Analysis over finite frequency domains}

In many practical applications performance specifications are only required over finite frequency ranges of interest., especially since commonly used reference signals. This paper therefore considers the case when (10) is modified to

$$
\left\|G_{c}(j \omega)\right\|_{\infty}<1, \forall \omega \in \Omega
$$

where $\Omega$ denotes the dominant finite frequency ranges, and $|\omega| \leq \varpi_{l}, \varpi_{1} \leq \omega \leq \varpi_{2},|\omega| \geq \varpi_{h}$ represent the low frequency range, middle frequency range and high frequency range, respectively.
Introduce the following notation

$$
\begin{aligned}
\Gamma_{1} & =\left[\begin{array}{ccc}
A & A_{d} & B_{0} \\
I & 0 & 0
\end{array}\right], \Gamma_{2}=\left[\begin{array}{ccc}
C & C_{d} & D_{0} \\
0 & 0 & I
\end{array}\right], \\
\Theta & =\left[\begin{array}{ccc}
X-\bar{d}^{-1} Z & \bar{d}^{-1} Z & 0 \\
\bar{d}^{-1} Z & -X-\bar{d}^{-1} Z & 0 \\
0 & 0 & 0
\end{array}\right] .
\end{aligned}
$$

The next result is Theorem 1 of [10] specialized to the single delay case and gives an LMI-based sufficient condition, which is dependent on the upper bounds of time-delay, for (10) to hold.

Lemma 5: For a given real symmetric matrix $\Pi$ of compatible dimensions and any delay $d$ satisfying $0<d \leq$ $\bar{d}$, a transfer-function matrix $G_{c}(j \omega)$ defined by (6) satisfies (10) if there exist $P \succ 0, Z \succ 0, Q \succ 0$ and a symmetric matrix $X$, such that

$$
\Gamma_{1}^{T}\left(\Phi \otimes P+\Psi \otimes Q+\Psi_{0} \otimes \bar{d} Z\right) \Gamma_{1}+\Gamma_{2}^{T} \Pi \Gamma_{2}+\Theta \prec 0,
$$

holds. Moreover the following frequency domain inequality also holds

$$
\left[\begin{array}{c}
G_{c}(j \omega) \\
I
\end{array}\right]^{T} \Pi\left[\begin{array}{c}
G_{c}(j \omega) \\
I
\end{array}\right] \prec 0, \forall \omega \in \Omega,
$$

where

$$
\begin{aligned}
& \Phi=\left[\begin{array}{ll}
0 & 1 \\
1 & 0
\end{array}\right], \Psi_{0}=\left[\begin{array}{ll}
1 & 0 \\
0 & 0
\end{array}\right], 0<d \leq \bar{d}
\end{aligned}
$$

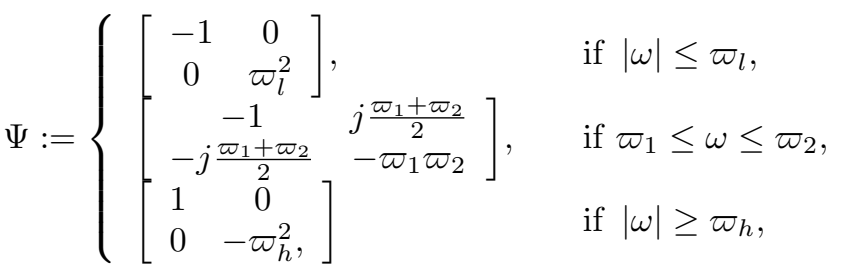

and the frequency range $\Omega$ is specified by $\Psi$.

Remark 2: The above Lemma requires the matrices $P$ and $X$ to be positive definite to guarantee that all eigenvalues of the matrix $\left(A+e^{-d j \omega} A_{d}\right)$ have strictly negative real parts as in [10]. This means that the condition $i i$ ) of Lemma 3 is immediately satisfied.

The inequality conditions in Lemma 5 are not convex and hence cannot be solved by using numerical software (e.g. Matlab LMi Control Toolbox) directly. To make this problem convex, the following transformations are used. Firstly, by Lemma 5, if

$$
\Pi=\left[\begin{array}{cc}
I & 0 \\
0 & -I
\end{array}\right]
$$

is used in (12) then (13) implies that (9) is satisfies for different frequency ranges as these defined by the matrix $\Psi$. Next, partition the matrix $\Xi$ as

$$
\Xi=\Phi \otimes P+\Psi \otimes Q+\Psi_{0} \otimes \bar{d} Z=\left[\begin{array}{c|c}
\Xi_{1} & \Xi_{2} \\
\hline(\star) & \Xi_{3}
\end{array}\right],
$$

where $\Xi$ takes different form according to the considered frequency range. In particular 
- for the low frequency range $\left(|\omega| \leq \varpi_{l}\right)$

$$
\Xi=\left[\begin{array}{c|c}
\Xi_{1} & \Xi_{2} \\
\hline(\star) & \Xi_{3}
\end{array}\right]=\left[\begin{array}{c|c}
-Q+\bar{d} Z & P \\
\hline(\star) & \varpi_{l}^{2} Q
\end{array}\right],
$$

- for the middle frequency range $\left(\varpi_{1} \leq \omega \leq \varpi_{2}\right)$

$$
\Xi=\left[\begin{array}{c|c}
\Xi_{1} & \Xi_{2} \\
\hline(\star) & \Xi_{3}
\end{array}\right]=\left[\begin{array}{c|c}
-Q+\bar{d} Z & P+j \frac{\varpi_{1}+\varpi_{2}}{2} Q \\
\hline(\star) & -\varpi_{1} \varpi_{2} Q
\end{array}\right],
$$

- and for the high frequency range $\left(|\omega| \geq \varpi_{h}\right)$

$$
\Xi=\left[\begin{array}{c|c}
\Xi_{1} & \Xi_{2} \\
\hline(\star) & \Xi_{3}
\end{array}\right]=\left[\begin{array}{c|c}
Q+\bar{d} Z & P \\
\hline(\star) & -\varpi_{h}^{2} Q
\end{array}\right] .
$$

Also, introduce

$$
Y=\left[\begin{array}{llll}
I & 0 & 0 & 0 \\
0 & I & 0 & 0
\end{array}\right]
$$

and

$$
\Upsilon=Y\left(\Phi \otimes P+\Psi \otimes Q+\Psi_{0} \otimes \bar{d} Z\right) Y^{T}+\Gamma_{2}^{T} \Pi \Gamma_{2}+\Theta,
$$

to rewrite the inequality of (12) as

$$
\left[\begin{array}{ccc}
A & A_{d} & B_{0} \\
I & 0 & 0 \\
0 & I & 0 \\
0 & 0 & I
\end{array}\right]^{T} \Upsilon\left[\begin{array}{ccc}
A & A_{d} & B_{0} \\
I & 0 & 0 \\
0 & I & 0 \\
0 & 0 & I
\end{array}\right] \prec 0 .
$$

This last inequality is of the form of the first of those in (1) when

$$
\Lambda^{\perp}=\left[\begin{array}{ccc}
A & A_{d} & B_{0} \\
I & 0 & 0 \\
0 & I & 0 \\
0 & 0 & I
\end{array}\right]
$$

and

$$
\Lambda=\left[\begin{array}{llll}
-I & A & A_{d} & B_{0}
\end{array}\right] .
$$

To use the result of Lemma 1 it is required to find $\Sigma^{\perp}$ that satisfies the second inequality of (1). Choosing

$$
\Sigma=\left[\begin{array}{llll}
\beta I & I & 0 & 0
\end{array}\right],
$$

gives

$$
\Sigma^{\perp}=\left[\begin{array}{ccc}
I & 0 & 0 \\
-\beta I & 0 & 0 \\
0 & I & 0 \\
0 & 0 & I
\end{array}\right]
$$

where $\beta$ is a given scalar and the desired formulations for low/middle/high frequency range are obtained by selecting $\beta$ as:

- $\beta=0$ for low and middle frequency range,

- $\beta>0$ for high frequency range.

$\mathrm{T}$ he above analysis introduces a slightly different formulation for the high frequency range since $\Xi_{1}$ in (17) cannot be negative definite because $Q \succ 0$ and $Z \succ 0$. Also with the $\Sigma$ and $\Sigma^{\perp}$ given above, the second inequality in (1) becomes

$$
\left[\begin{array}{ccc}
\Sigma_{11} & -\beta C^{T} C_{d}-\beta \bar{d} Z & -\beta C^{T} D_{0} \\
(\star) & C_{d}^{T} C_{d}-X-\bar{d}^{-1} Z & C_{d}^{T} D_{0} \\
(\star) & (\star) & D_{0}^{T} D_{0}-I
\end{array}\right] \prec 0,
$$

where

$$
\Sigma_{11}=\Xi_{1}-\beta^{2}\left(\Xi_{3}-C^{T} C-X+\bar{d} Z\right)-\beta\left(\Xi_{2}+\Xi_{2}^{T}\right) .
$$

The first two new results in this paper can now be derived where, unlike Theorem 1 in [10], the high frequency range is considered separately since it requires $\beta>0(\operatorname{not} \beta=0)$ and hence the resulting LMI has a different form than for low and middle frequency ranges.

\section{B. Main results}

The following theorem guarantees stability along the pass of the differential repetitive processes considered over finite frequency specifications. In this result, the matrices $\Xi_{1}$, $\Xi_{2}, \Xi_{3}$ form a matrix $\Xi$ of the form (14) and are chosen according to the specific frequency range, i.e. for low, middle or high frequency range, respectively, as given in the previous section.

Theorem 1: A differential linear repetitive process described by (4) is stable along the pass and the finite frequency performance specifications over low and middle frequency ranges given in (13) are met for any delay $d$ satisfying $0<d \leq \bar{d}$ if there exist matrices $\hat{P} \succ 0, \hat{Q} \succ 0, \hat{X} \succ 0$, $\hat{Z} \succ 0$ and $S$ such that

$$
\left[\begin{array}{ccccc}
\hat{\Xi}_{1} & \hat{\Xi}_{2}-S^{T} & 0 & 0 & 0 \\
(\star) & \mathrm{T}_{1} & A_{d} S+\bar{d}^{-1} \hat{Z} & B_{0} & (C S)^{T} \\
(\star) & (\star) & -\hat{X}-\bar{d}^{-1} \hat{Z} & 0 & \left(C_{d} S\right)^{T} \\
(\star) & (\star) & (\star) & -I & D_{0}^{T} \\
(\star) & (\star) & (\star) & (\star) & -I
\end{array}\right] \prec 0,
$$

where $\mathrm{T}_{1}=\hat{\Xi}_{3}+\hat{X}-\bar{d}^{-1} \hat{Z}+\operatorname{sym}\{A S\}, \hat{\Xi}_{1}=-\hat{Q}+\bar{d} \hat{Z}$, and

$$
\begin{aligned}
& \hat{\Xi}_{2}=\hat{P}, \hat{\Xi}_{3}=\varpi_{l}^{2} \hat{Q}, \text { for low frequency range } \\
& \hat{\Xi}_{2}=\hat{P}+j \frac{\varpi_{1}+\varpi_{2}}{2} \hat{Q}, \hat{\Xi}_{3}=-\varpi_{1} \varpi_{2} \hat{Q}, \text { for middle frequency range. }
\end{aligned}
$$

Proof: Suppose that the LMI (21) holds. Then, preand post-multiplying (21) by $\operatorname{diag}\left\{S^{-T}, S^{-T}, S^{-T}, I, I\right\}$ and its transpose, respectively, gives

$$
\left[\begin{array}{ccccc}
\Xi_{1} & \Xi_{2}-W & 0 & 0 & 0 \\
(\star) & \tilde{\Xi}_{3} & \bar{d}^{-1} Z+W^{T} A_{d} & W^{T} B_{0} & C^{T} \\
(\star) & (\star) & -X-\bar{d}^{-1} Z & 0 & C_{d}^{T} \\
(\star) & (\star) & (\star) & -I & D_{0}^{T} \\
(\star) & (\star) & (\star) & (\star) & -I
\end{array}\right] \prec 0
$$

where

$\tilde{\Xi}_{3}=\Xi_{3}+X-\bar{d}^{-1} Z+\operatorname{sym}\left\{A^{T} W\right\}, W=S^{-1}, P=S^{-T} \hat{P} S^{-1}$,

$\Xi_{1}=S^{-T} \hat{\Xi}_{1} S^{-1}, \Xi_{2}=S^{-T} \hat{\Xi}_{2} S^{-1}, Q=S^{-T} \hat{Q} S^{-1}$

$\Xi_{3}=S^{-T} \hat{\Xi}_{3} S^{-1}, X=S^{-T} \hat{X} S^{-1}, Z=S^{-T} \hat{Z} S^{-1}$.

Another application of the Schur's complement formula gives that (23) holds if and only if

$$
\left[\begin{array}{cccc}
\Xi_{1} & \Xi_{2}-W & 0 & 0 \\
(\star) & \tilde{\Xi}_{3}+C^{T} C & \Gamma_{3} & C^{T} D_{0}+W^{T} B_{0} \\
(\star) & (\star) & \Gamma_{4} & C_{d}^{T} D_{0} \\
(\star) & (\star) & (\star) & D_{0}^{T} D_{0}-I
\end{array}\right] \prec 0,
$$

where

$$
\Gamma_{3}=C^{T} C_{d}+\bar{d}^{-1} Z+W^{T} A_{d}, \Gamma_{4}=C_{d}^{T} C_{d}-X-\bar{d}^{-1} Z .
$$


It is immediate feasibility of this last inequality implies that $D_{0}^{T} D_{0}-I \prec 0$ and therefore condition $i$ ) of Lemma 3 must hold. Moreover, the last inequality can be rewritten as

$$
\Upsilon+\operatorname{sym}\left\{\Lambda^{T} W \Sigma\right\} \prec 0,
$$

where $\Upsilon$ is defined in (18) and

$$
\Lambda=\left[\begin{array}{llll}
-I & A & A_{d} & B_{0}
\end{array}\right], \Sigma=\left[\begin{array}{llll}
0 & I & 0 & 0
\end{array}\right] .
$$

Also $\Sigma=\left[\begin{array}{llll}0 & I & 0 & 0\end{array}\right]$ is obtained by setting $\beta=0$ in (20). Therefore, by Lemma 1 it follows that (24) is feasible if and only if (12) holds. Hence conditions $i i$ ) and $i i i)$ of Lemma 3 must hold and the proof is complete.

Selecting $\beta>0$ and using a similar approach gives the following result for stability along the pass in the highfrequency range.

Theorem 2: With the notation of (22), a differential linear repetitive process described by (4) is stable along the pass and the finite frequency performance specifications over high frequency ranges given in (13) are met for any delay $d$ satisfying $0<d \leq \bar{d}$ if there exist matrices $\hat{P} \succ 0, \hat{Q} \succ 0$, $\hat{X} \succ 0, \hat{Z} \succ 0, S$, and a positive scalar $\beta$ such that

$$
\left[\begin{array}{ccccc}
\Gamma_{5} & \Gamma_{6} & \beta A_{d} S & \beta B_{0} & 0 \\
(\star) & \mathrm{T}_{1} & A_{d} S+\bar{d}^{-1} \hat{Z} & B_{0} & (C S)^{T} \\
(\star) & (\star) & -\hat{X}-\bar{d}^{-1} \hat{Z} & 0 & \left(C_{d} S\right)^{T} \\
(\star) & (\star) & (\star) & -I & D_{0}^{T} \\
(\star) & (\star) & (\star) & (\star) & -I
\end{array}\right] \prec 0,
$$

where

$$
\Gamma_{5}=\hat{\Xi}_{1}-\beta S-\beta S^{T}, \Gamma_{6}=\hat{\Xi}_{2}-S^{T}+A S .
$$

Proof: This follows from routine changes to the proof of the previous result to account for $\beta>0$. Hence the details are omitted.

\section{A frequency-partitioned approach for stability along the pass}

Divide the entire frequency range, i.e., from $\omega=0$ to $\omega=\infty$, into $H$ intervals (not necessarily containing the same number of frequencies) such that

$$
[0, \infty)=\bigcup_{h=1}^{H}\left[\omega_{h-1}, \omega_{h}\right],
$$

where $\omega_{0}=0$ and $\omega_{H}=\infty$. Then the LMI conditions in Theorems 1 and 2, respectively, can be applied over these frequency intervals. In particular, the control performance for low frequency range is imposed over the first interval, i.e. $h=1$ and those for the high frequency range over the last interval, i.e. $h=H$. The specifications for the middle frequency range can be defined over remaining intervals for $2 \leq h \leq H-1$. Furthermore, the LMI condition in Theorems 1 and 2 guarantee that $\bar{\sigma}\left(G_{c}(j \omega)\right)<1$ (where $G_{c}(j \omega)$ is defined in (6)) over the prescribed frequency ranges. However, some practical control specifications require that $\bar{\sigma}\left(G_{c}(j \omega)\right)<\mu$ where $0<\mu \leq 1$, i.e., a prescribed rate of pass-to-pass convergence over some frequencies is required.

\section{CONTROLler Design}

In this section, the LMI based stability conditions developed in the previous section are extended to the problem of controller design. The control law to be applied has the following form over $0 \leq t \leq \alpha, k \geq 0$

$$
u_{k+1}(t)=K_{1} x_{k+1}(t)+K_{2} y_{k}(t),
$$

where $K_{1}$ and $K_{2}$ are matrices of compatible dimensions to be designed. This control law uses feedback of the current pass state vector (which is assumed to be available for feedback) and 'feedforward' of pass profile vector from the previous pass. It is important to note that the term 'feedforward' is used to describe the case where state or pass profile information from the previous pass (or passes) is used as (part of) the input to a control law applied on the current pass, i.e. to information which is propagated in the pass to pass $(k)$ direction.

This form of control law has an obvious physical meaning for repetitive processes considered in this paper and the following result holds.

Theorem 3: Suppose that the control law of (27) is applied to a differential repetitive process of the form (4). Then the resulting process is stable along the pass and the finite frequency performance specifications over low and middle frequency ranges given in (13) and any delay $d$ satisfying $0<d \leq \bar{d}$ if there exist matrices $\hat{P} \succ 0, \hat{Q} \succ 0, \hat{X} \succ 0$, $\hat{Z} \succ 0, S, N$ and $K_{2}$ such that

$$
\left[\begin{array}{ccccc}
\hat{\Xi}_{1} & \hat{\Xi}_{2}-S^{T} & 0 & 0 & 0 \\
(\star) & \Gamma_{7} & \Gamma_{8} & B_{0}+B K_{2} & (C S+D N)^{T} \\
(\star) & (\star) & \Gamma_{9} & 0 & \left(C_{d} S\right)^{T} \\
(\star) & (\star) & (\star) & -I & \left(D_{0}+D K_{2}\right)^{T} \\
(\star) & (\star) & (\star) & (\star) & -I
\end{array}\right] \prec 0
$$

where

$$
\begin{aligned}
& \Gamma_{7}=\hat{\Xi}_{3}+\hat{X}-\bar{d}^{-1} \hat{Z}+\operatorname{sym}\{A S+B N\} \\
& \Gamma_{8}=A_{d} S+\bar{d}^{-1} \hat{Z}, \Gamma_{9}=-\hat{X}-\bar{d}^{-1} \hat{Z},
\end{aligned}
$$

and where the notation introduced in (22) is used. Moreover, if the above LMI is feasible, the corresponding matrices in the control law (27) are $K_{2}$ (directly computed during solving LMI of (28)) and $K_{1}$ is computed as $K_{1}=N S^{-1}$.

Proof: Assume that the control law of (27) is applied to a differential repetitive process described by (4), resulting in the following state- space model for the controlled process

$$
\begin{aligned}
\dot{x}_{k+1}(t)= & \left(A+B K_{1}\right) x_{k+1}(t)+A_{d} x_{k+1}(t-d) \\
& +\left(B_{0}+B K_{2}\right) y_{k}(t), \\
y_{k+1}(t)= & \left(A+D K_{1}\right) x_{k+1}(t)+C_{d} x_{k+1}(t-d) \\
& +\left(D_{0}+D K_{2}\right) y_{k}(t) .
\end{aligned}
$$

Next, routine matrix manipulations show that the above process is stable along the pass as setting $N=K S$ allows the application of the result of Theorem 1. This transformation leads directly to LMI of (28) and the proof is complete.

The result holds for the high frequency range.

Theorem 4: Suppose that the control law of (27) is applied to a differential repetitive process of the form (4). Then 
the resulting process is stable along the pass and the finite frequency performance specifications over high frequency ranges given in (13) are met for any delay $d$ satisfying $0<d \leq \bar{d}$ if there exist matrices $\hat{P} \succ 0, \hat{Q} \succ 0, \hat{X} \succ 0$, $\hat{Z} \succ 0, S, N$ and a positive scalar $\beta$ such that

$$
\left[\begin{array}{ccccc}
\Gamma_{10} & \Gamma_{11} & \beta A_{d} S & \beta\left(B_{0}+B K_{2}\right) & 0 \\
(\star) & \Gamma_{7} & \Gamma_{8} & B_{0}+B K_{2} & (C S+D N)^{T} \\
(\star) & (\star) & \Gamma_{9} & 0 & \left(C_{d} S\right)^{T} \\
(\star) & (\star) & (\star) & -I & \left(D_{0}+D K_{2}\right)^{T} \\
(\star) & (\star) & (\star) & (\star) & -I
\end{array}\right] \prec 0
$$

where $\Gamma_{10}=\hat{\Xi}_{1}-\beta S-\beta S^{T}, \Gamma_{11}=\hat{\Xi}_{2}-S^{T}+A S+B N$ and where the notation introduced in (22) is used. Moreover, if the above LMI is feasible, the corresponding matrices in the control law (27) are computed the same as for Theorem 3.

Proof: This result is proved, with routine changes, in the same way as Theorem 3. Hence the details are omitted.

\section{Simulation Study}

To illustrate the effectiveness of the proposed design procedure the numerical example is provided. Let us consider the particular case of (4) when

$A=\left[\begin{array}{cc}-0.005 & -5.8 \\ 1 & -0.005\end{array}\right], A_{d}=\left[\begin{array}{cc}-0.005 & -0.8 \\ 0 & -0.001\end{array}\right], D_{0}=1.2$,
$B=\left[\begin{array}{l}0.1 \\ 0.5\end{array}\right], B_{0}=\left[\begin{array}{l}0.1 \\ 0.5\end{array}\right], C=\left[\begin{array}{ll}1 & 0\end{array}\right], C_{d}=\left[\begin{array}{ll}0.1 & 0\end{array}\right], D=0.8$

This example is asymptotically unstable and hence unstable along the pass since $D_{0}>1$, i.e., condition $i$ ) of Lemma 3 is not satisfied. Executing the design procedure given in Section IV for $\bar{d}=0.4$ and the following frequency ranges

$$
[0,3] \cup[3,15] \cup[15, \infty],
$$

gives the following control law matrices for $\beta=0.2$

$$
K_{1}=[-1.3328-2.1505], K_{2}=-1.4523
$$

It can be easily verified that the resulting controlled process is stable along the pass since $\rho\left(D_{0}+D K_{2}\right)=0.0382$, and it can be verified that $\rho\left(G_{c l}(\mathrm{j} \omega)\right)<1$ for all $\omega$, where $G_{c l}(\mathrm{j} \omega)$ is given by

$$
\begin{aligned}
G_{c l}(j \omega)= & \left(C+D K_{1}+e^{-d j \omega} C_{d}\right)\left(j \omega I-A-B K_{1}-e^{-d j \omega} A_{d}\right)^{-1} \\
& \times\left(B_{0}+B K_{2}\right)+\left(D_{0}+D K_{2}\right) .
\end{aligned}
$$

This can be seen in Figure 1 and this plot confirms that the design specifications are met.

\section{CONCLUSION}

This paper has developed results that enable finite frequency domain specification to be imposed in the stability analysis and control law design for differential linear repetitive processes with time-delays. It has been shown that the delay-dependent stability condition for these processes can be reformulated as a convex optimization procedure in the form of an LMI. These are the first results in this area and much further work remains to be done. For example, it is necessary to investigate the trade-offs between using this

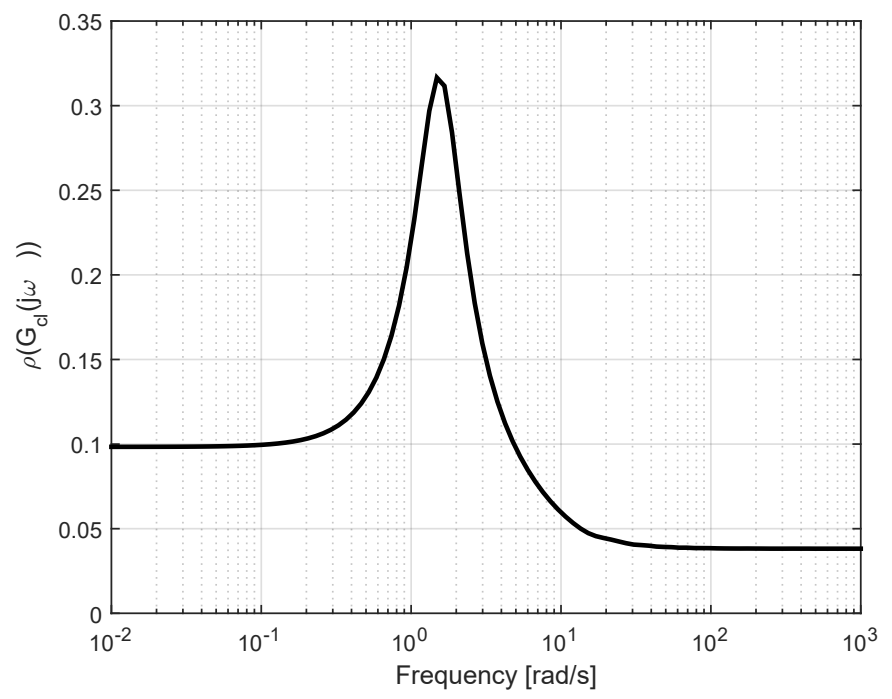

Fig. 1. Plot of $\rho\left(G_{c l}(\mathrm{j} \omega)\right.$.

approach and alternative schemes which use only measured outputs in the form of the pass profile vectors but at the (possible) expense of requiring dynamics in the control law itself.

\section{ACKNOWLEDGMENTS}

The authors gratefully acknowledge the contribution of National Science Centre in Poland, grant No. 2014/15/B/ST7/03208

\section{REFERENCES}

[1] E. Rogers and D. H. Owens, Stability Analysis for Linear Repetitive Processes, ser. Lecture Notes in Control and Information Sciences. Berlin, Germany: Springer-Verlag, 1992, vol. 175.

[2] E. Rogers, K. Gałkowski, and D. H. Owens, Control Systems Theory and Applications for Linear Repetitive Processes, ser. Lecture Notes in Control and Information Sciences. Berlin, Germany: Springer-Verlag, 2007, vol. 349.

[3] W. Paszke, E. Rogers, and K. Gałkowski, "Experimentally verified generalized kyp lemma based iterative learning control design," Control Engineering Practice, vol. 53, no. 10, pp. 57-67, 2016.

[4] P. D. Roberts, "Two-dimensional analysis of an iterative nonlinear optimal control algorithm," IEEE Transactions on Circuits and Systems I: Fundamental Theory and Applications, vol. 49, no. 6, pp. 872-878, 2002.

[5] C. Briat, Linear Parameter-Varying and Time-Delay Systems. Analysis, Observation, Filtering \& Control, ser. Advances in Delays and Dynamics. Heidelberg, Germany: Springer-Verlag, 2015, vol. 3.

[6] T. Iwasaki and S. Hara, "Generalized KYP lemma: unified frequency domain inequalities with design applications," IEEE Transactions on Automatic Control, vol. 50, no. 1, pp. 41-59, 2005.

[7] P. Gahinet and P. Apkarian, "A linear matrix inequality approach to $\mathcal{H}_{\infty}$ control," International Journal of Robust and Nonlinear Control, vol. 4, pp. 421-448, 1994.

[8] H. Tao, W. Paszke, E. Rogers, H. Yang, and K. Gałkowski, "Iterative learning fault-tolerant control for differential time-delay batch processes in finite frequency domains," Journal of Process Control, vol. 56, no. Supplement C, pp. 112-128, 2017.

[9] G. Pipeleers and L. Vandenberghe, "Generalized KYP lemma with real data," IEEE Transactions on Automatic Control, vol. 56, no. 12, pp. 2942-2946, 2011.

[10] X. Zhang and G. Yang, "Performance analysis for multi-delay systems in finite frequency domains," International Journal of Robust and Nonlinear Control, vol. 22, no. 8, pp. 933-944, 2012. 